\title{
Developing an Autonomous Behavior of Virtual Users Based on Psychological Interpretation of Human Behavior to an Atypical Architectural Shape
}

\author{
Young Lim Lee ${ }^{1}$, Yun Gil Lee ${ }^{2 *}$ \\ ${ }^{1}$ Assistant Professor, Department of Psychology \& Psychotherapy, Dankook \\ University, 119 Dandae-ro Dongnam-gu, Cheonan-si, Chungcheongnam-do, \\ Republic of Korea \\ ${ }^{2}$ Associate Professor, Department of Architecture, Hoseo University, 20 Hoseo-ro \\ 79beon-gil, Baebang-eup, Asan-si, Chungcheongnam-do, Republic of Korea

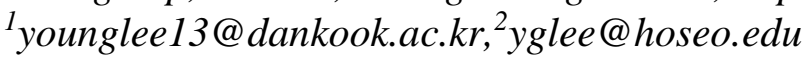

\begin{abstract}
Virtual users (VUsers) enable analytical and observable representations in human behavior simulations. By utilizing advanced, intelligent VUsers, architects can discover new inspirations for designs or problems that could be overlooked. This study describes a way to enhance VUsers' intelligence, focusing on VUsers' responses to atypical architectural spaces. In our previous research, we investigated users' behavior in the atypical architecture spaces and the psychological causes of this behavior. In this study, we focus on computerization and systematic integration of our prior results in order to develop technology to automatically set the place where user behavior can be expressed in an atypical architectural design and VUsers can exhibit psychologically appropriate behavior. For this purpose, we investigated how to designate trigger spots, trigger viewpoints, and behavioral areas, which are necessary for the autonomous behavior of VUsers. The results of this research will be integrated into ActoViz, a user behavior simulation system.
\end{abstract}

Keywords: Behavior Simulation, Expert System, Intelligent Agent, Virtual User, Atypical Architectural Design

\section{Introduction}

Virtual users (VUsers) are agents that can calculate the physical and semantic properties of unique attributes, behavioral rules, sets of goals, and built environments and automatically provide analytical and observable representations of the expected behavioral responses [10]. By utilizing advanced, intelligent VUsers, architects can discover new inspiration for designs or problems that could be overlooked. This study aims to enhance VUsers' intelligence, focusing on VUsers' responses to atypical architectural spaces [11][12]. In our previous research, we investigated users' behavior in atypical architecture spaces and the psychological causes of this behavior. In this study, we perform computerization and systematic integration

Article history:

Received (May 6, 2019), Review Result (June 2, 2019), Accepted (August 28, 2019)

* Corresponding author: Yun Gil Lee, Associate Professor, Department of Architecture, Hoseo University, 20

Hoseo-ro 79beon-gil, Baebang-eup, Asan-si, Chungcheongnam-do, Republic of Korea, yglee@hoseo.edu 
of these results, developing a technology that automatically sets the place where users' behavior can be expressed in an atypical architectural design and that features VUsers exhibiting psychologically appropriate behavior. For this purpose, this study investigated how to designate a trigger spot, trigger viewpoint, and behavioral area, which are necessary for VUsers to behave autonomously. The results of this research will be integrated into ActoViz, a user behavior simulation system.

\section{Human Behavior in Atypical Architecture and Psychological In- terpretations}

The previous study analyzed the characteristics of human users' behavior in an atypical architectural space. Atypical architectural elements induced user movement through the temporal stimulation, and users performed specific actions regarding physical elements such as inclination and height. By analyzing this user behavior and psychologically interpreting them, we enhance the intelligence of VUsers and develop a more meaningful simulation system.

\subsection{Behavior regarding handrails}

Gibson (1962) suggested that active touch tends to relate to the object being contacted; the texture of the surface and the dynamism of the form or the degree to which it is graspable determine one's desire to touch it. In our context, the information users receive from their active touch senses and their desire to touch a handrail determine whether they grab it when going down a set of stairs [1][2].

\subsection{Looking down over a railing}

Freud stated that we have a certain amount of libido ("desire" in Latin), a type of mental energy, and if we suppress an impulse to do a forbidden action, this energy appears in a different form. Not everyone suppresses these impulses; for example, when evaluating situations in which safety is ensured (e.g., the height of a staircase), some may look down [3].

\subsection{Leaning on a tilted wall or railing}

A tilted structure appears dynamic, which can lead users to want to move or lean as well. Also, as it is more difficult to perceive oblique orientations than horizontal or the vertical orientations, one can use haptic information to compensate when exploring the tilted structure $[2][4][5]$.

\subsection{Moving up curved stairs}

Gibson believed that perceptual attributes that can be used to immediately control a particular behavior are more important than spatial attributes, such as the size, distance, or form of objects in a three-dimensional space, which are emphasized in the traditional approach. For a person who wants to jump over a small creek, a perceptual attribute is not the width of the creek itself, but whether the creek is narrow enough to jump over. To do this, we use the optic flow produced when we move, which provides real-time information about where we are heading and how fast we are moving. In an atypical architectural space, users might use optic flow when they move up a curved staircase [6][7][8][9]. 


\subsection{Children jumping up stairs}

Perception is an act of intention. When we perceive an object, we perceive both its physical properties (i.e., what the object is) and its meaning (i.e., how it can be used). These two types of attributes cannot be separated. Since the affordance of an object is related to its meaning, it can differ for different users. A child may view a staircase and think of whether they can jump up it, while an adult may view the staircase as walkable. In this way, perception and action cannot be separated; one's perception of a situation generates action, and changes to the situation as a result of the action generate new perceptions [1][6].
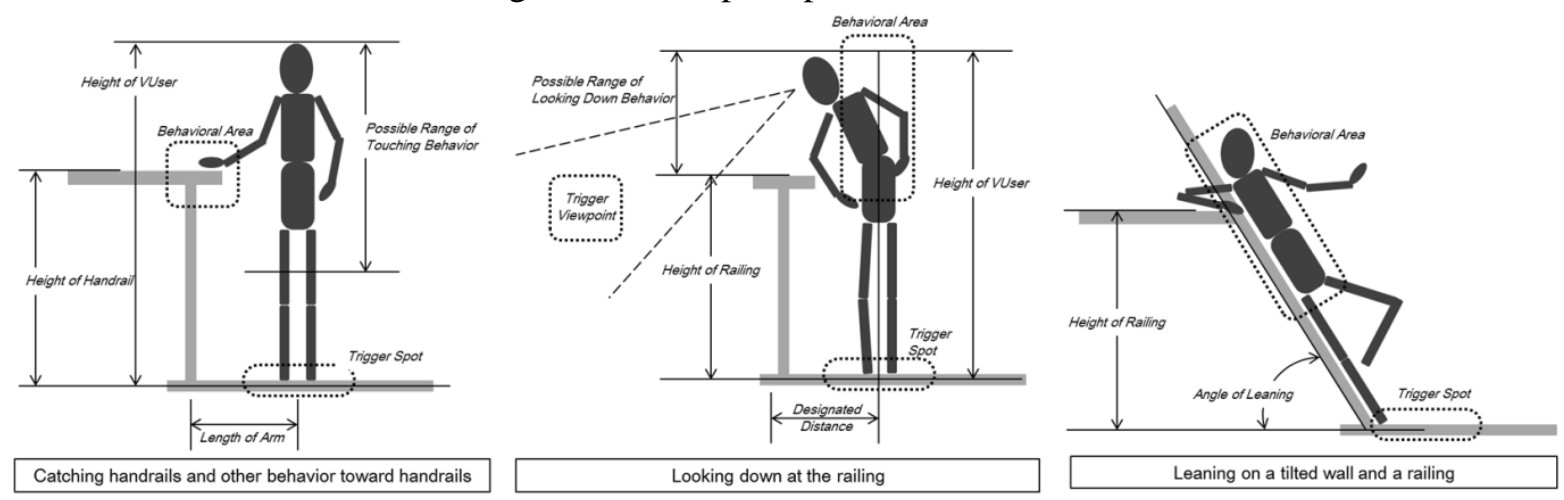

Figure 1. Specifying trigger spots and behavioral areas according to the type of action performed by VUsers

\section{Trigger Spot, Trigger Viewpoint, and Behavioral Area for Autonomous Behavior}

To develop VUsers that automatically perform psychologically appropriate actions in an unstructured architectural design, we must determine, for example, how to assign the trigger spot, how to get VUsers to visit the trigger spot, and how to get VUsers to exhibit responsive behavior at the trigger spot.

We can assign the trigger spot to be automatically derived from an atypical architectural model. Such a model involves innumerable geometrical faces, each of which is positioned and oriented in a particular way. In general, atypical buildings feature inclined faces or unusual angles at which faces meet. In other words, the edges described in the height of the VUsers or those where two slanted faces meet are places where VUsers are likely to exhibit behavior. In this study, we call these behavioral areas. The foot positions that VUsers adopt when performing proper behaviors are referred to as trigger spots. Behavioral areas and trigger spots are defined separately because they offer different advantages for finding paths and modeling behavior. According to field surveys, the location of the trigger spot differs somewhat depending on the type of each activity. Figure 1 shows methods of specifying a trigger spot and behavioral area according to the type of action performed by a VUser. For examples of these actions, we use touching a handrail, looking down over a railing, and leaning on a tilted wall (see sections 2.1, 2.2, and 2.3).

If there are things to see nearby, such as a stairway, it is necessary to set the trigger viewpoint. Figure 2 shows how one can set the trigger spot and trigger viewpoint on an outer part of a staircase (see sections 2.4 and 2.5). Walking up the inside of curved stairs has psychological and economical interpretations. In addition, VUsers' behavior can be influenced by the 
tendency of other VUsers and the environment. Thus, trigger spots and trigger viewpoints can be set based on VUsers' tendencies.

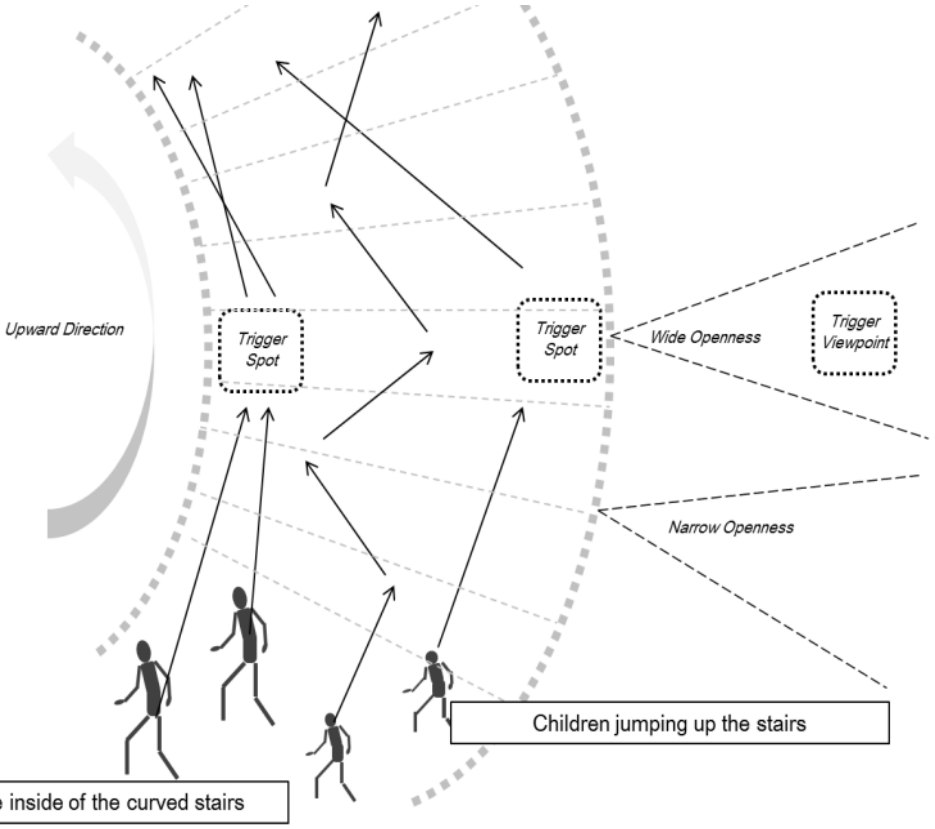

Figure 2. Specifying a trigger spot and trigger viewpoint on stairs

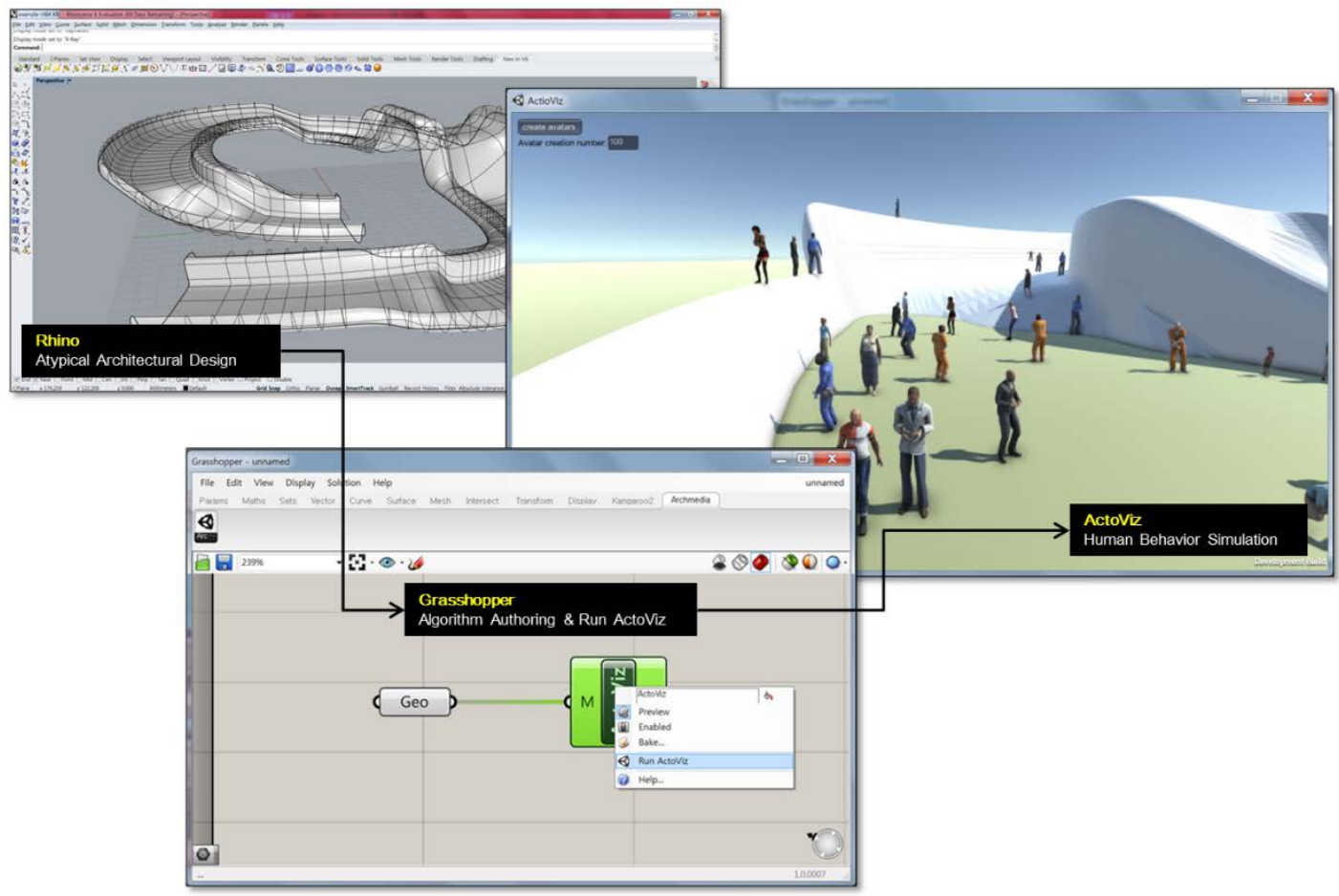

Figure 3. Process of executing ActoViz using Rhino and Grasshopper

Figure 3 shows the process of running ActoViz, an add-on module for Grasshopper, which is frequently used for designing atypical buildings. The module is a type of export system that 
can simulate human behaviors using VUsers while taking into account the geometry of the design. The VUsers act freely and behave in a variety of different ways in the simulation. Figure 4 shows how the trigger spot, trigger viewpoint, and behavioral area can be specified in ActoViz. Application of this information to an atypical architectural space enhances the autonomy of VUsers, enabling them to act appropriately in a psychologically meaningful location.

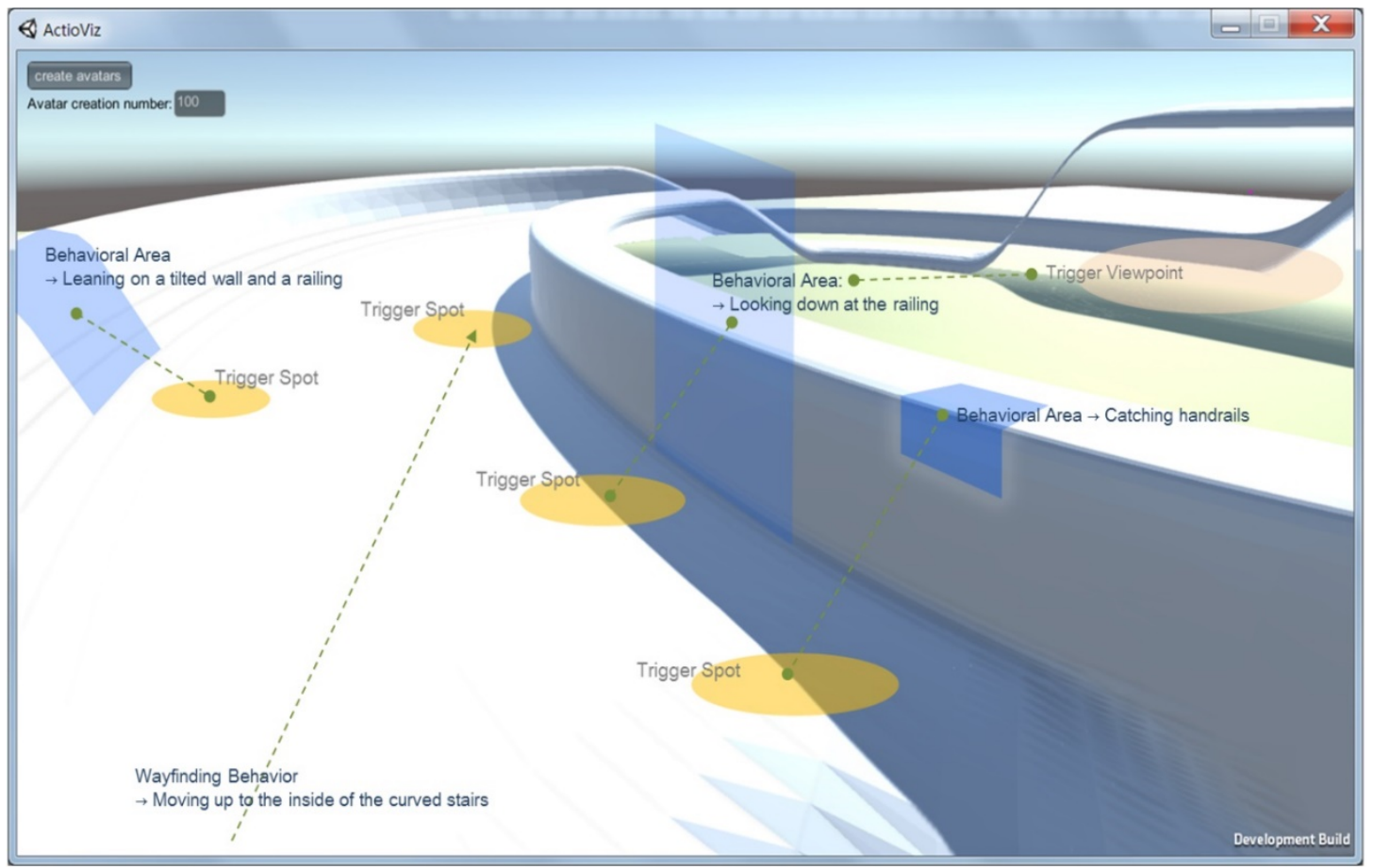

Figure 4. Methods of specifying the trigger spot, trigger viewpoint, and behavioral area using ActoViz

\section{Conclusion and discussion}

This study aims to develop a technology that automatically sets places where user behavior can be expressed in atypical architectural design and in which VUsers exhibit psychologically appropriate behavior. Primarily, this paper describes how to arrange and use trigger spots, trigger viewpoints, and behavioral areas to enhance the intelligence and autonomy of VUsers in atypical architectural spaces. Algorithms for how such information can be automatically derived from ActoViz and how VUsers may use such information need to be developed in further research. However, the results of this research are significant, as they can be used in relation to autonomous driving methods, physics, and artificial intelligence and aid the development of more natural and meaningful simulations.

\section{Acknowledgements}

This work was supported by the National Research Foundation of Korea (NRF) grant funded by the Korea government (MSIT) (NRF-2018R1A2B6005827). 
Developing an Autonomous Behavior of Virtual Users Based on Psychological Interpretation of Human Behavior to an Atypical Architectural Shape

\section{References}

[1] J. J. Gibson, Observations on active touch. Psychological Review. 69(6), pp.477-491 (1962)

[2] B. E. Goldstrin, Sensation and Perception, 9th edition. Cengage Learning (2013)

[3] R. Schönhammer, Einführung in die Wahrnehmungspsychologie: Sinne, Körper, Bewegung, UTB GmbH (2013)

[4] R. Arnheim, A plea for visual thinking. Critical Inquiry, 6(3), pp.489-497 (1980)

[5] Y. Lee, C. E. Crabtree, J. F. Norman, G. P. Bingham, Poor shape perception is the reason reaches-to-grasp are visually guided online. Perception \& Psychophysics, 70(6), pp.1032-1046 (2008)

[6] J. J. Gibson, The ecological approach to visual perception: classic edition. Psychology Press (2014)

[7] J. W. Philbeck, J. M. Loomis, Comparison of two indicators of perceived egocentric distance under full-cue and reduced-cue conditions. Journal of Experimental Psychology: Human Perception and Performance, 23(1), pp.72-85 (1997)

[8] J. T. Todd, J. F. Norman, The visual perception of 3-D shape from multiple cues: Are observers capable of perceiving metric shape? Perception \& Psychophyscis, 65(1), pp.31-47 (2003)

[9] Land, M., Lee, D.: Where we look when we steer. Nature, 369, 742-744 (1994)

[10] Y. E. Kalay, Architecture's new media: Principles, theories, and methods of computer-aided design. Cambridge, MA: MIT Press (2004)

[11] A. Jalalian, S. K. Chalup M. J. Ostwald, Architectural evaluation of simulated pedestrian spatial behaviour. Architectural Science Review, 54(2), pp.132-140 (2011)

[12] D. Simeone, Y. E. Kalay, D. Schaumann, S. Hong, Modeling and simulating use processes in buildings. In 2013 Proceedings of Education and Research in Computed Aided Architectural Design in Europe (pp. 59-66). Delft, Netherlands: Education and Research in Computer Aided Architectural Design in Europe (2013) 